The purpose of this Brief Communications section is to present important research results of more limited scope than regular articles appearing in Physics of Fluids. Submission of material of a peripheral or cursory nature is strongly discouraged. Brief Communications cannot exceed three printed pages in length, including space allowed for title, figures, tables, references, and an abstract limited to about 100 words.

\title{
Onset and end of drop formation along the surface of turbulent liquid jets in still gases
}

\author{
P.-K. Wu ${ }^{a)}$ and G. M. Faeth \\ Department of Aerospace Engineering, The University of Michigan, Ann Arbor, Michigan 48109-2118
}

(Received 25 April 1995; accepted 3 July 1995)

\begin{abstract}
The onset and end of drop formation along the surface of turbulent liquid jets in still gases was studied for liquid/gas density ratios greater than 500 where aerodynamic effects are small. Measurements were correlated using a phenomenological turbulent breakup theory based on the interactions between the turbulence energy spectrum and the energy requirements for drop formation. The onset and end of drop formation along the surface generally was associated with turbulent eddies in the internal and large-eddy subranges of the turbulence spectrum, respectively, although breakup of the entire liquid column ends breakup along the surface in some instances, as well. () 1995 American Institute of Physics.
\end{abstract}

Drop formation along the surface of liquid jets in still gases is an important fundamental process relevant to liquid jet atomization. Earlier work has considered the onset of drop formation and the variation of drop sizes with distance from the jet exit for turbulent liquid jets, successfully correlating these properties based on a phenomenological turbulent breakup theory. ${ }^{1=3} \mathrm{New}$ measurements and phenomenological analysis were undertaken during the present studyemphasizing processes causing drop formation to end-in order to gain a better understanding of drop formation along the surface of turbulent liquid jets in still gases.

The test apparatus was the same as in previous works, ${ }^{1-4}$ and involved vertically downward injection of various liquids in still air at normal temperature and pressure. The liquid injectors had rounded inlets followed by straight tubes, with tube length/diameter ratios, $L / d \geqslant 40$, in order to ensure fully-developed turbulent pipe flow at the jet exit. ${ }^{1-4}$ Pulsed shadowgraphy was used for flow visualization and measurements. Measurements were averaged over five observations to yield experimental uncertainties (95\% confidence) less than $25 \%$, dominated by the statistical variations of drop and ligament separation from the liquid surface. Results considered include present measurements, as well as earlier data for similar conditions, ${ }^{1,2,5,6}$ yielding Weber numbers $\left(\mathrm{We}_{f d}=\rho_{f} d u_{0}^{2} / \sigma\right)$ of $1.0 \times 10^{2}-1.1 \times 10^{6}$, Reynolds numbers $\left(\mathrm{Re}_{f d}=\rho_{f} d u_{0} / \mu_{f}\right)$ of $3.4 \times 10^{3}-8.5 \times 10^{5}$ and Ohnesorge numbers $\left(\mathrm{OH}_{d}=\mu_{f} /\left(\rho_{f} d \sigma\right)^{1 / 2}\right)$ of $0.001-0.017$, where $\rho_{f}$ is the liquid density, $u_{0}$ is the average liquid velocity at the jet exit, $\sigma$ is the surface tension of the liquid, and $\mu_{f}$ is the viscosity of the liquid. Aerodynamic effects were small over the test range because liquid/gas density ratios were larger than $500 .^{3}$

Figures $1-3$ are shadowgraphs illustrating the general

${ }^{a)}$ Present address: Taitech Inc., AMC P. O. Box 33830, WPAFB, Ohio 45433-0830. nature of drop formation for present test conditions. The positions noted in the figure captions refer to the distance, $x$, between the injector exit and the tip of the reference pin visible in each shadowgraph. At the lowest $\mathrm{We}_{f d}$, Fig. 1, the liquid column does not generate drops until it breaks up as a whole; this behavior is conventionally called first windinduced breakup. ${ }^{4,7}$ Different behavior is observed at larger $\mathrm{We}_{f d}$, Fig. 2, where drop formation from the tips of ligaments starts along the liquid surface at $x / d \approx 10$, and the liquid column is surrounded by drops at larger distances from the injector exit; however, careful study of surface ligament behavior shows that drop formation along the liquid surface actually stops again at $x / d \approx 66$ which is before the liquid column breaks up as a whole. Finally, a third behavior is observed at even higher values of $\mathrm{We}_{f d}$, Fig. 3, where drop formation from the tips of ligaments starts along the liquid surface at $x / d \approx 5$, and careful study of surface ligament behavior shows that this type of surface breakup persists over the whole liquid column (and even continues from the surface of the large ligaments formed by liquid column breakup). Naturally, once drop formation starts along the liquid surface, the liquid column is shrouded by drops to some extent, even when drop formation along the surface subsequently stops; therefore, the behavior illustrated in both Figs. 2 and 3 is conventionally called second wind-induced breakup regime. ${ }^{4,7}$

The phenomena discussed in connection with Figs. 1-3 can be explained by extending the earlier turbulent breakup theory of Wu et al..$^{1,2}$ to consider the large-eddy subrange of the turbulence spectrum, and combining these results with earlier liquid core length findings for turbulent liquid jets in still gases. ${ }^{1,5,6}$ Present considerations are limited to conditions where aerodynamic effects are small; therefore, the main hypothesis of the turbulent breakup theory is that drops are formed from turbulent eddies when the kinetic energy of radial fluctuations of the eddy is larger than the surface en- 


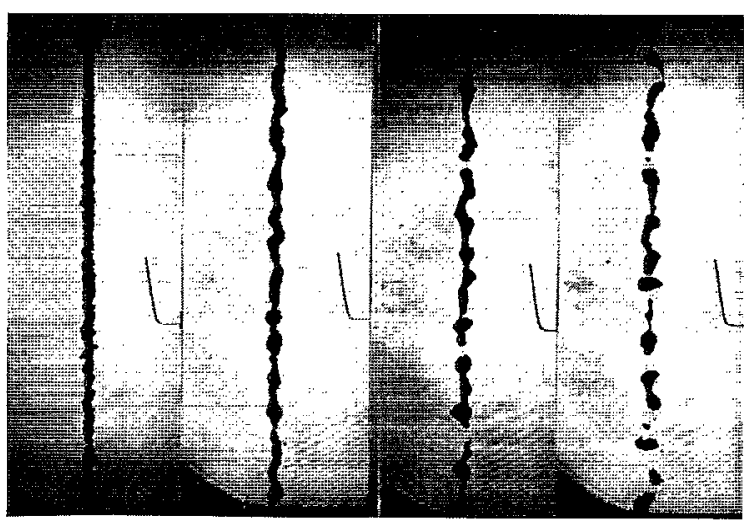

FIC. 1. Shadowgraphs of a turbulent water jet in still air at $\mathrm{We}_{f d}=4940$ and (left to right) $x / d=20,70,120$, and 170 .

ergy required to form a drop of corresponding size. ${ }^{1,2}$ This yields the following criterion for drop formation:

$$
\rho_{f} v_{l}^{2}\left(\pi l^{3} / 6\right) / 2 \geq C_{l}\left(4 \pi l^{2}\right) \sigma
$$

where $l$ is the characteristic eddy size, $v_{l}$ is the radial fluctuation velocity corresponding to $l$, and $C_{l}$ is an empirical factor of order of magnitude unity to account for various geometrical and scaling factors. Drop sizes observed over the test range were significantly larger than the Kolmogorov length scale; therefore, subsequent considerations will be limited to turbulent eddies in the large-eddy and inertial subranges (although drop sizes after turbulent breakup comparable to the Kolmogorov length scale can be encountered in some instances and which merits attention). Then, the radial fluctuation velocity can be estimated as $v^{2} \sim k E(k)$, where $k$ is the wave number and $E(k)$ is the three-dimensional energy spectrum which exhibits slopes of 1 and $-5 / 3$ in the large-eddy and inertial subranges. ${ }^{1,8}$ For present purposes, the transition between these two regimes will be ignured and the spectrum will be approximated by straight lines following the power law fits for the large-eddy and inertial subranges, illustrated in Fig. 4. Given these approximations, $v_{l}$ can be related to the radial root-mean-square fluctuating velocity, $v^{\prime}$, and the radial integral scale, $\Lambda$, as follows:

$$
v_{l} / v^{\prime} \sim(l / \Lambda)^{n},
$$

where $n=-1$ and $1 / 3$ for the large-eddy and inertial subranges, respectively. The range of drop sizes that can be observed as a result of turbulent breakup can then be found by

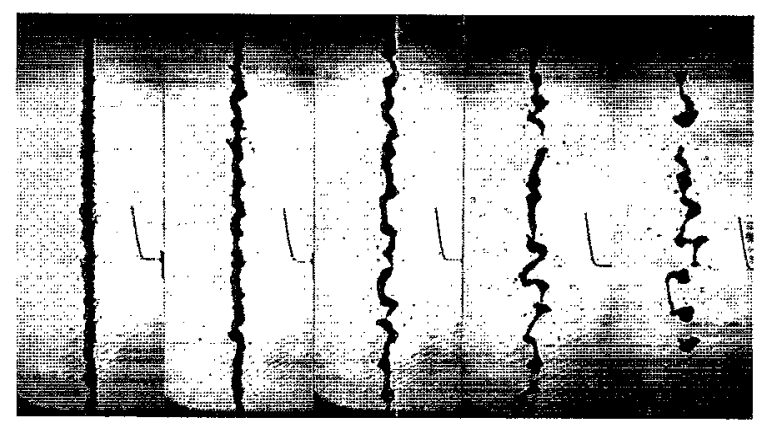

FIG. 2. Shadowgraphs of a turbulent water jet in still air at $\mathrm{We}_{f d}=11920$ and (left to right) $x / d=20,75,150,200$, and 250 .

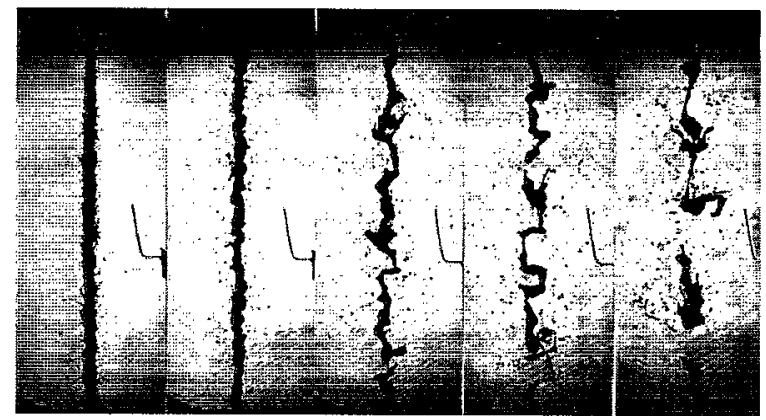

FIG. 3. Shadowgraphs of a turbulent water jet in still air at $\mathrm{We}_{f d}=20280$ and (left to right) $x / d=20,70,170,230$, and 255 .

substituting Eq. (2) into Eq. (1) and assuming that $l$ is proportional to the Sauter mean diameter, SMD, of the spray, as follows:

$$
C_{i s} \mathrm{We}_{f d}^{-0.6} \leqslant \mathrm{SMD} / d \leqslant C_{e s} \mathrm{We}_{f d},
$$

where $C_{i s}$ and $C_{e s}$ are empirical constants associated with drop sizes at the onset and end of turbulent breakup along the surface. Notably, the onset drop size of Eq. (3) was in reasonably good agreement with the observations of Wu et al. ${ }^{1}$ yielding $C_{i s}=2.37$.

The location of the onset and end of drop formation along the liquid surface can be found by estimating the time and distance from the jet exit that is required to form a drop of a given size, $l$. It was assumed that the time required to form a drop, $\tau$, was proportional to the Rayleigh breakup time scale of an eddy of size $l$, i.e., $\tau$ $\sim\left(\rho_{f} l^{3} / \sigma\right)^{1 / 2}$, see Wu et al. ${ }^{1}$ In the same way, it also was assumed that the growing disturbances at the surface of the turbulent liquid were convected with the mean streamwise injection velocity so that the distance from the jet exit to the breakup location, $x \sim \tau u_{0}$. Combining these ideas with Eq. (3) yields

$$
C_{i x} \mathrm{We}_{f d}^{-0.4} \leqslant x / d \leqslant C_{e x} \mathrm{We}_{f d}^{2},
$$

where $C_{i x}$ and $C_{e x}$ are empirical constants associated with the locations of the onset and end of turbulent breakup, $x_{i}$ and $x_{e}$, along the surface.

The feasibility of drop formation at the maximum energy condition of the turbulence spectrum is an important aspect of breakup along the liquid surface. From Fig. 4 the maximum energy condition occurs at $l=l_{e}$ where $l_{e} / \Lambda=4.3$ and the characteristic eddy velocity at $l_{e}$ is $v_{l e} / v^{\prime}=1.6{ }^{8}$ Introducing these factors into Eq. (1), then yields a critical Weber number for no turbulent breakup along the liquid surface of $\mathrm{We}_{f d}^{*}=2450$.

Another interesting limit on conditions for drop formation along the surface of turbulent liquid jets is provided by the length of the liquid core, $L_{c}$. This length can be estimated by noting that breakup of the liquid core occurs when drop sizes formed by breakup along the liquid surface are comparable to the diameter of the liquid core itself, when aerodynamic effects are small, as follows: ${ }^{1}$

$$
L_{c} / d=C_{c} \mathrm{We}_{f d}^{\mathrm{i} / 2}
$$




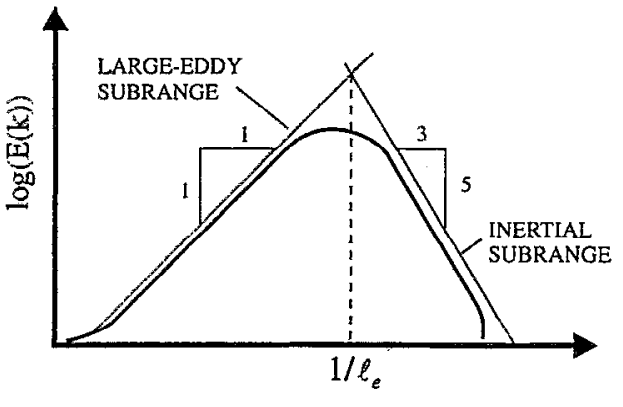

$\log (\mathrm{k}$ or $1 / \mathrm{l})$

FIG. 4. Sketch of three-dimensional turbulent energy spectrum.

where $C_{c}$ is an empirical constant of order unity. Grant and Middleman ${ }^{5}$ and Chen and Davis ${ }^{6}$ measured $L_{c}$ for turbulent liquid jets in still gases, when aerodynamic effects were small, also finding $L_{c}$ along the lines of Eq. (5).

The discussion of Eqs. (4) and (5) suggests that the onset and end of turbulent breakup along the liquid surface, and the length of the liquid core, all correlate in terms of $\mathrm{We}_{f d}$. Thus present measurements of these properties, as well as the earlier results of $\mathrm{Wu}$ and coworkers, ${ }^{1-3}$ Grant and Middleman $^{5}$ and Chen and Davis ${ }^{6}$ are plotted as suggested by Eqs. (4) and (5) in Fig. 5 . It is evident that fair correlations of $x_{i} / d, x_{e} / d$, and $L_{c} / d$ can be obtained in this manner. The expression for the location of the onset of turbulent breakup along the surface is

$$
x_{i} / d=2000 \mathrm{We}_{f d}^{-0.67}
$$

with standard deviations of the coefficient and power of $10 \%$ and $12 \%$, respectively, and the correlation coefficient of the fit equal to 0.91 . The large value of the coefficient of Eq. (6) comes about because it is proportional to $\left(u_{0} / v_{0}^{\prime}\right)^{9 / 5}$, which is a large number of turbulent pipe flow. ${ }^{1}$ The expression for the end of turbulent breakup along the surface is

$$
x_{e} / d=1.58 \times 10^{-5} \mathrm{We}_{f d}^{1.68}
$$

with standard deviations of the constant and power of $16 \%$ and $11 \%$, respectively, and the correlation coefficient of the fit equal to 0.94. Finally, the expression for $L_{c}$ is

$$
L_{c} / d=7.40 \mathrm{We}_{f d}^{0.34}
$$

with standard deviations of the constant and power of $4 \%$ and $3 \%$, respectively, and the correlation coefficient of the fit equal to 0.98 . The differences between the powers suggested by Eqs. (4) and (5) and the powers found from the empirical fits of Eqs. (6)-(8) are statistically significant but are not large in view of the approximations of the theory and the uncertainties of the measurements.

The correlations for $x_{i}$ and $x_{e} / d$ divide the flow into surface and no surface breakup regimes, and intersect each other at $\mathrm{We}_{f d}^{*} \approx 2800$, which is close to the earlier prediction of $\mathrm{We}_{f d}^{*}=2450$. The measurements yield a higher value, $\mathrm{We}_{f d}^{*} \approx 5200$, with the smaller predicted value resulting from the simplified power law approximation of the energy spectrum illustrated in Fig. 4. For $\mathrm{We}_{f d} \leqslant 5200$, the jets do not undergo surface breakup which corresponds to the conventional first wind-induced breakup regime., ${ }^{4,7}$ For 5200 $\leqslant \mathrm{We}_{f d} \leqslant 17000$, the liquid jet exhibits both the onset and end

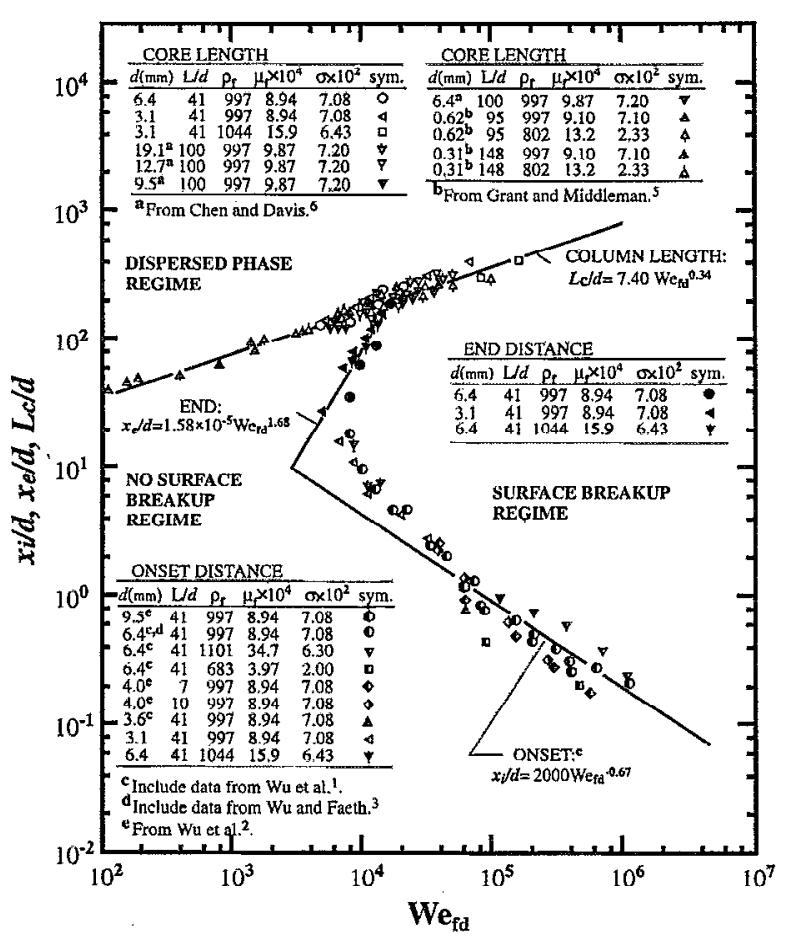

FIG. 5. Surface breakup regime map for turbulent liquid jets in still gases when aerodynamic effects are small.

of surface breakup before the liquid column breaks up as a whole. Finally, for $\mathrm{We}_{f d} \geqslant 17000$, surface breakup continues beyond the end of the liquid column. For moderate values of $\mathrm{We}_{f d} \geqslant 5200$, the onset of surface breakup begins at some distance from the jet exit and the flow would be described as second wind-induced breakup. ${ }^{4,7}$ With increased $\mathrm{We}_{f d}$, however, turbulent liquid surface brcakup begins progressively closer to jet exit, eventually reaching the criterion for the atomization breakup regime where surface breakup occurs close to the injector exit. ${ }^{4,7}$

\section{ACKNOWLEDGMENTS}

This research was sponsored by the Office of Naval Research Grant Nos. N00014-89-J-1199 and N00014-95-10234 under the technical management of G. D. Roy and E. P. Rood, respectively, and by the Air Force Office of Scientific Research Grant No. AFOSR-92-1321 under the technical management of J. M. Tishkoff.

${ }^{1}$ P.-K. Wu, L-K. Tseng, and G. M. Faeth, "Primary breakup in gas/liquid mixing layers for turbulent liquids," Atom. Sprays 2, 295 (1992).

${ }^{2}$ P.-K. Wu, R. F. Miranda, and G. M. Faeth, "Effects of initial flow conditions on primary breakup of nonturbulent and turbulent liquid jets," Atom Sprays 5, 175 (1995).

${ }^{3}$ P.-K. Wu and G. M. Faeth, "Aerodynamic effects in primary breakup of turbulent liquids," Atom. Sprays 3, 265 (1993).

${ }^{4}$ G. A. Ruff, A. D. Sagar, and G. M. Faeth, "Structure of the near-injector region of pressure-atomized sprays," ALAA J. 27, 901 (1989).

${ }^{5}$ R. P. Grant and S. Middleman, "Newtonian jet stability," AIChE J. 12, 669 (1966).

${ }^{6}$ T.-F. Chen and J. R. Davis, "Disintegration of a turbulent water jet," J. Hydraulic Dívision, Proc. ASCE 90, HY1, 175 (1964).

${ }^{7}$ R. D. Reitz, "Atomization and other breakup regimes of a liquid jet," Ph.D, thesis, Princeton University, p. 134, 1978.

${ }^{8}$ J. O. Hinze, Turbulence, 2nd ed. (McGraw-Hill, New York, 1975), pp. 202-259 and 724-742. 